\title{
NANOMETER LIGHT SOURCE AND MOLECULAR EXCITON MICROSCOPY
}

\author{
R. KOPELMAN ${ }^{\mathrm{a}}$, A. LEWIS ${ }^{\mathrm{b}}$ and K. LIEBERMAN ${ }^{\mathrm{b}}$ \\ ${ }^{a}$ Department of Chemistry, University of Michigan, Ann Arbor, MI 48109, USA \\ ${ }^{b}$ Department of Applied Physics, Hebrew University, Jerusalem, Israel
}

\begin{abstract}
We describe the development of a new nanometer light source based on exciton transmitted optical radiation as well as a new biologically non-invasive ultraresolution light microscopy, based on combining the energy transfer "spectral ruler" method with the micro-movement technology employed in STM. We use near-field scanning optical microscopy, with micropipettes containing crystals of energy packaging donor molecules in the tips that can have apertures below $5 \mathrm{~nm}$. The excitation of these tips extends near field microscopy well beyond the $50 \mathrm{~nm}$ limit. The theoretical resolution limit for this spectrally sensitive light microscope is well below $1 \mathrm{~nm}$. The $Z$-distance feedback control is illustrated by the exciton-plasmon transfer from an anthracene crystal tip to a thin gold film (transparent to light).
\end{abstract}

\section{Introduction}

The near-field scanning optical microscope (NSOM), developed by Lewis and co-workers, has enabled researchers to optically examine a variety of specimens without being limited in resolution to one half a wavelength of light [1,2]. All NSOM imaging is based on the fact that as an electromagnetic wave emerges from an aperture it is at first highly collimated to the aperture dimension. It is only after the wave has propagated a finite distance from the aperture that the diffraction which limits classical optical imaging takes effect. In the near-field region a beam of light exists that is largely independent of the wavelength and determined solely by the size and shape of the aperture [4].

The most succesful results to date have been obtained using a metal coated glass micropipette as an aperture [2]. Pipettes can readily be produced with inner diameters at the tip of several hundred angstroms [3]. Their tapered profile is almost ideal for probing recessed regions of non-planar surfaces. The metallic coating serves both to confine the light to the dimension of the pipette and as a conducting probe where tunneling feedback is employed. The details of pipette fabrication have been described elsewhere [4].

The current resolution limitation of the NSOM technique derives from the fact that the intensity falls off exponentially with the aperture cross section. The solution to this problem is to use the energy packaging capabilities of molecular excitons [5]. By growing a suitable crystal within the submicron confines of a pipette, energy can be guided directly to the aperture at the tip instead of being allowed to propagate freely in the form of an electromagnetic wave. Such a material can be excited through an electrical or radiative process to produce an abundance of excitons that allows light to be effectively propagated through the bottleneck created by the subwavelength dimensions of the tip near the aperture. The excitons can be excited directly at the tip or they can be generated within the bulk of the material and allowed to diffuse to the tip via an excitonic transfer $[5,6]$. In either case, in a suitable material these excitons will then undergo a radiative decay producing a tiny source of light at the very tip of the pipette. The excitonic throughput is basically independent of the wavelength and falls off only linearly with the cross-section of the aperture.

\section{Experimental procedure}

The crystal of anthracene was grown inside the very tip of a pipette from a benzene solution. By varying the concentration of the solution the size of the deposited crystal could be accurately controlled.

The source of excitation for our experiments was the $3638 \AA$ line of an argon ion laser. Anthracene exhibits a very strong fluorescence in the blue with a quantum efficiency approaching unity when illuminated in the near UV. The crystal can be illuminated either by directing light through the pipette as in the standard aperturing method, or alternatively by having an external beam incident on the crystal at the tip of the pipette. With the second method large amounts of energy can be brought to bear directly on the spot where the illumination is desired, with the upper limit being imposed only by the onset of photochemical bleaching. A fairly large $(\sim 1 \mu \mathrm{m})$ crystal was grown extending past the tip of the pipette and illuminated, head on, with several $\mathrm{mW}$ at $3638 \AA$. The spot of light produced was clearly visible to the naked eye. 


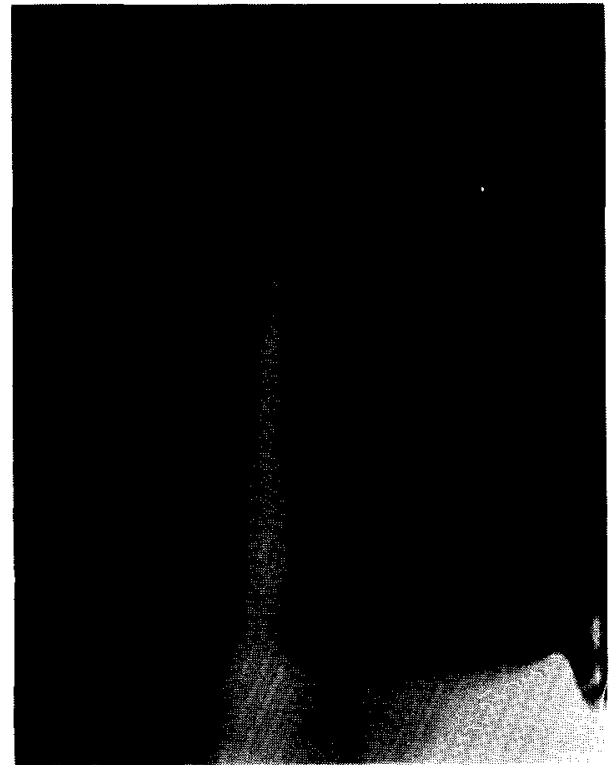

Fig. 1. Micropipette exciton light source with anthracene crystal.

The test sample was a $2 \mu \mathrm{m}$ aluminum grating. The imaging was done in the transmission mode with the excitatory beam being directed through the pipette. A filter was placed behind the grating to prevent any stray UV light from reaching the photomultiplier, confining the signal to the crystal fluorescence. The lifetime of the singlet state in anthracene, responsible for most of the

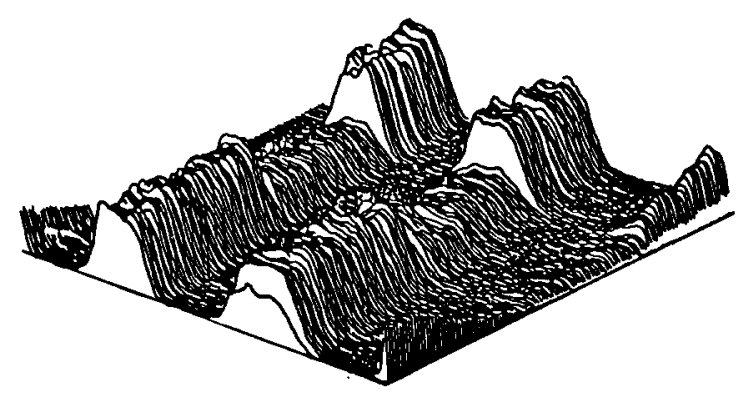

Fig. 2. Scan of aluminum grating with dust particle $(20 \mathrm{~min}$ scan, $9 \times 9 \mu \mathrm{m}$ ). luminescence, is sufficiently short (about $10 \mathrm{~ns}$ ) to allow the beam to be chopped and a lock-in detection scheme to be employed.

\section{Results and discussion}

The result of a single line scan $9 \mu \mathrm{m}$ long and a complete two-dimensional scan is shown in fig. 2 . The image is unprocessed and the resolution could be further improved by deconvoluting the image with the well-defined shape of the pipette aperture.

For the smallest pipette experiment calibrated so far (ID $60 \mathrm{~nm}$ ), the improvement in light transmission via the excitonic process is at least $150 \%$. Extrapolating to smaller aperture dimensions, the improvement at $6 \mathrm{~nm}$ should be about a factor of $10^{13}$ and the throughput about $10^{4}$ photons/s. Nanometer scanning optical microscopy has thus become a reality.

\section{Acknowledgements}

Research supported by the Innovative Science and Technology Office of the Strategic Defense Initiative through a grant to AL administered by the Office of Naval Research. RK's research on excitons is supported by National Science Foundation grant DMR-8801120.

\section{References}

[1] A. Lewis et al., Ultramicroscopy 13 (1984) 227.

[2] E. Betzig, A. Harootunian, A. Lewis and M. Isaacson, Appl. Opt. 25 (1986) 1890

[3] A. McDonald, IEEE Trans. Microwave Theory Tech. MTT-20 (1972), 689.

[4] T. Brown and D.G. Flaming, Neuroscience 2 (1977) 813.

[5] M. Pope and E. Swenberg, Electronic Processes in Organic Crystals (Oxford Univ. Press, New York, 1982).

[6] A.H. Francis and R. Kopelman, in: Laser Spectroscopy of Solids, eds. W.M. Yen and P.M. Seltzer, Topics in Appl. Phys. Vol. 49, 2nd ed. (Springer, Berlin, 1986) p. 241. 